\title{
Feedback Stabilization with Discrete Measurements using Bounds on Fundamental Matrices
}

\author{
Frédéric Mazenc and Michael Malisoff
}

\begin{abstract}
We prove a new robust stabilization theorem for systems with time-varying disturbances and sampled measurements, using novel bounds on fundamental matrices for systems with disturbances. Our main tools use properties of positive systems and Metzler matrices. Our numerical example illustrates an advantage of using our method instead of previous methods.
\end{abstract}

\section{INTRODUCTION}

The study of time-varying systems [12] is frequently rendered difficult by the fact that in general, no formula for state transition (i.e., fundamental solution) matrices can be obtained, and estimating them may not be easy. This problem often arises when the vector fields defining the systems are uncertain, and where one must also find a globally asymptotically stabilizing feedback control that can be computed from only sampled state measurements. While such sampling problems can be recast as delay compensating problems where the delay is a timevarying sawtooth shaped function, delay compensation methods normally require exact knowledge, or close approximations, of the vector fields defining the dynamics [4], or they can lead to bounds on the allowable delays (and so also bounds on the allowable sample rates) that might not always be large enough for applications. See, e.g., [7], [8], and [15] for motivation for studying the effects of sampling in control engineering.

To help address these challenges, this note provides a new construction of functions that are composed of exponential functions which upper and lower bound the entries of the state transition matrix of a linear system with a poorly known but bounded vector field, where the inequalities are componentwise. We apply this result to build robust stabilizing controls for linear systems with discrete measurements. Our control formula is the one from [6, Chapter 2, Section 2.9], which is applied in [6] to cases where the state is available for continuous measurement. Here, we apply the approach from [6] to systems whose variables are only measured at discrete instants. This lets us cover cases where the sup norm of

Key Words: stabilization, sampling. Supported by NSF Grants 1711299 and 2009659 (Malisoff).

F. Mazenc is with Inria Saclay, L2S-CNRS-CentraleSupélec, 3 rue Joliot Curie, 91192, Gif-sur-Yvette, France (e-mail: frederic.mazenc@12s.centralesupelec.fr)

M. Malisoff is with Department of Mathematics, Louisiana State University, Baton Rouge, LA 70803, USA (e-mail: malisoff@1su.edu) the uncertainty in the dynamics and the sample rate are arbitrarily large, which we believe is a significant and novel contribution. Our proofs use comparison systems which are reminiscent of interval observers (as defined, e.g., in [5] and [11]), but are outside the scope of previous interval observers that did not consider sampling and unknown vector fields. We use novel applications of properties of Metzler matrices and positive systems. While positive systems have been used in aerospace engineering, mathematical biology, and other fields, we believe that our work is the first to apply them to sample data feedback stabilization under uncertain vector fields, where the sup norms of the uncertainty and the intersample intervals can be arbitrarily large.

In Section II, we provide the relevant definitions and notation. Then in Section III, we state our main feedback stabilization theorem. In Section IV, we state our new theorems on estimating fundamental matrix solutions of time-varying systems, which are of independent interest because of the importance of estimating fundamental matrices for uncertain systems, and which we use to prove our stabilization theorem in Section V. We illustrate our findings in Section VI by showing a potential advantage of our approach compared with earlier methods, and we close in Section VII by summarizing our contributions and suggesting future research problems.

\section{DEFINITIONS AND NotATION}

We use the following definitions and notation in the sequel, where the dimensions of our systems are arbitrary unless other indicated. The notation will be simplified whenever no confusion would arise given the context, and all of the matrices in this note are assumed to have only real entries. A matrix is called Metzler provided its off diagonal entries are all nonnegative. For two vectors $V_{1}=\left(v_{1,1} \ldots v_{1, n}\right)^{\top}$ and $V_{2}=\left(v_{2,1} \ldots v_{2, n}\right)^{\top}$, we write $V_{1}<V_{2}$ when for all $i \in\{1, \ldots, n\}, v_{1, i}<v_{2, i}$; and $V_{1} \leq V_{2}$ when for all $i \in\{1, \ldots, n\}, v_{1, i} \leq v_{2, i}$. We adopt similar notation for matrices. Let $\|\cdot\|$ denote the standard Euclidean norm of vectors and matrices. We define functions $\Xi_{t}$ by $\Xi_{t}(s)=\Xi(t+s)$ for all $\Xi, s$, and $t$ for which $t+s$ is in the domain of $\Xi$. We set $G^{+}=\left[\max \left\{g_{i j}, 0\right\}\right]$ for matrices $G=\left[g_{i j}\right]$ and $G^{-}=G^{+}-G$. Note for later use that if $M_{1}$ and $M_{2}$ are matrices of the same size such that $M_{1} \leq M_{2}$, 
then we have the ordering properties $M_{1}^{+} \leq M_{2}^{+}$and $M_{2}^{-} \leq M_{1}^{-}$. Let $I$ denote the identity matrix of any dimension, and let 0 denote the matrix of any dimensions whose entries are all equal to zero. Square matrices $M_{1}$ and $M_{2}$ of the same size are called similar provided there is an invertible matrix $P$ such that $M_{1}=P^{-1} M_{2} P$.

For a continuous function $\mathcal{F}:[0,+\infty) \rightarrow \mathbb{R}^{n \times n}$, the fundamental (or state transition) matrix solution $\Phi_{\mathcal{F}}$ is defined to be the unique matrix valued solution of

$$
\frac{\partial \Phi_{\mathcal{F}}}{\partial t}\left(t, t_{0}\right)=\mathcal{F}(t) \Phi_{\mathcal{F}}\left(t, t_{0}\right), \quad \Phi_{\mathcal{F}}\left(t_{0}, t_{0}\right)=I
$$

for all real $t$ and $t_{0}$. We also use basic properties of input-to-state stability (or ISS, which we also use to mean input-to-state stable), e.g., from [14].

\section{Stabilization Theorem}

We study systems of the form

$$
\dot{X}(t)=\left(M-\delta_{1}(t)\right) X(t)+B U(t)+\delta_{2}(t),
$$

(but see Remark 2 for more general systems that covered by our methods) with $X$ valued in $\mathbb{R}^{n}$, the control $U$ valued in $\mathbb{R}^{p}, M \in \mathbb{R}^{n \times n}$ and $B \in \mathbb{R}^{n \times p}$ being constant matrices, and $\delta_{1}:[0,+\infty) \rightarrow \mathbb{R}^{n \times n}$ and $\delta_{2}:[0,+\infty) \rightarrow \mathbb{R}^{n}$ being piecewise continuous and locally bounded. We assume that the available output measurement $Y$ is defined by $Y(t)=X\left(t_{i}\right)$ for all $t \in\left[t_{i}, t_{i+1}\right)$, where $t_{0}=0$ and the $t_{i}$ 's present times when new state measurements become available, and we define $\sigma:[0,+\infty) \rightarrow[0,+\infty)$ by $\sigma(t)=t_{i}$ for all $t \in\left[t_{i}, t_{i+1}\right)$. We assume the following (but see Remark 2 for non-Metzler cases):

Assumption 1: The matrix $M$ is Metzler, there is a matrix $\bar{\delta} \geq 0$ such that

$$
0 \leq \delta_{1}(t) \leq \bar{\delta}
$$

for all $t \geq 0$, the pair $(M, B)$ is controllable, $\inf _{i \geq 0}\left\{t_{i+1}-t_{i}\right\}>0$, and there is a known constant $\nu>0$ such that $\sup _{i>0}\left\{t_{i+1}-t_{i}\right\} \leq \nu$.

We then use the functions and matrices

$$
\begin{aligned}
& \bar{\xi}(\ell)=\frac{e^{(M+\bar{\delta}) \ell}+e^{(M-\bar{\delta}) \ell}}{2}-e^{M \ell}, \\
& \underline{\xi}(\ell)=\frac{e^{(M-\bar{\delta})}-e^{(M+\bar{\delta}) \ell}}{2}, S=B B^{\top}, \\
& \text { and } J(r)=\int_{0}^{r} e^{-M \ell} S e^{-M^{\top} \ell} \mathrm{d} \ell .
\end{aligned}
$$

Since $(M, B)$ is controllable, $J^{-1}$ is well-defined for all $r>0$, e.g., by [13, Corollary 3.2.10 and Theorem 5]. Then, for all $r \in(0, \nu]$, the matrices

$$
\begin{aligned}
\overline{\mathcal{F}}_{c}(r)= & \bar{\xi}(r)+\int_{0}^{r}\left[\bar{\xi}(r-\ell)\left(S e^{-M^{\top} \ell} J^{-1}(r)\right)^{-}\right. \\
& \left.-\underline{\xi}(r-\ell)\left(S e^{-M^{\top} \ell} J^{-1}(r)\right)^{+}\right] \mathrm{d} \ell \\
\underline{\mathcal{F}}_{c}(r)= & \underline{\xi}(r)+\int_{0}^{r}\left[\underline{\xi}(r-\ell)\left(S e^{-M^{\top} \ell} J^{-1}(r)\right)^{-}\right. \\
& \left.-\bar{\xi}(r-\ell)\left(S e^{-M^{\top} \ell} J^{-1}(r)\right)^{+}\right] \mathrm{d} \ell
\end{aligned}
$$

are well-defined. Using the matrices (5), our stabilization theorem is as follows, where we use the entrywise supremum notation $\sup _{r \in[0, \nu]} \mathcal{M}(r)=\left[\sup _{r \in[0, \nu]} \mathcal{M}_{i j}(r)\right]$ for matrix valued functions $\mathcal{M}(r)=\left[\mathcal{M}_{i j}(r)\right]$ :

Theorem 1: Let the system (2) satisfy Assumption 1. Then (2), in closed-loop with the control defined by

$$
U(t)=-B^{\top} e^{M^{\top}\left(t_{i}-t\right)} J^{-1}\left(t_{i+1}-t_{i}\right) X\left(t_{i}\right)
$$

for all $t \in\left[t_{i}, t_{i+1}\right)$ and $i \geq 0$, is ISS with respect to $\delta_{2}$ if the matrix

$$
\Gamma=\sup _{r \in(0, \nu]}\left[\begin{array}{ll}
\overline{\mathcal{F}}_{c}^{+}(r) & \underline{\mathcal{F}}_{c}^{-}(r) \\
\underline{\mathcal{F}}_{c}^{-}(r) & \overline{\mathcal{F}}_{c}^{+}(r)
\end{array}\right]
$$

is Schur stable.

Before providing our fundamental matrix estimation theorems (and then our proof of Theorem 1 in Section $\mathrm{V})$, we discuss the novelty and value of Theorem 1.

Remark 1: A crucial aspect of Theorem 1 is that one can check whether $\Gamma$ is Schur stable because the matrices $\overline{\mathcal{F}}_{c}(r)$ and $\overline{\mathcal{F}}_{c}(r)$ are expressed in terms of known functions, whereas the state transition matrix of $M-\delta_{1}(t)$ cannot be found since $\delta_{1}$ is unknown. In the special case where $\delta_{2}$ is the zero function, our proof of Theorem 1 will show that the origin of the closed-loop system is a globally exponentially stable equilibrium on $\mathbb{R}^{n}$. For ways to compute $\overline{\mathcal{F}}_{c}$ and $\overline{\mathcal{F}}_{c}$, see $[1, \mathrm{p}$.34].

Remark 2: By Theorem 3 from Section IV-B, we can extend Theorem 1 to cases where $M$ is not Metzler but is similar to a Metzler matrix, by first using a change of coordinates to transform (2). We can also cover cases where $M$ and $B$ are time varying, with added uncertainty also on $B$ [10]. The control (6) is the one of [6, Chapter 2, Section 2.9]. We can find a matrix $\delta_{B}>0$ so that $\Gamma$ is Schur stable when $0 \leq \bar{\delta} \leq \delta_{B}$ since $\underline{\xi}$ and $\bar{\xi}$ are continuous and zero when $\bar{\delta}=0$. This facilitates checking the assumptions of Theorem 1 .

\section{Estimation of Fundamental Matrices}

In this section, we state our theorems on estimating fundamental matrices, which are of independent interest. In Section V, we will see how these estimation theorems can be used to prove Theorem 1 .

\section{A. Metzler Case}

We first address the problem of estimating fundamental solutions, using a known Metzler term in the vector field defining the system; see Section IV-B for a way to relax this Metzler requirement. Throughout this subsection, we fix a $\Delta$ that satisfies:

Assumption 2: The function $\Delta:[0,+\infty) \rightarrow \mathbb{R}^{n \times n}$ is a piecewise continuous matrix valued function. Also, there is a known constant nonnegative matrix $\bar{\Delta} \in \mathbb{R}^{n \times n}$ such that $0 \leq \Delta(t) \leq \bar{\Delta}$ for all $t \geq 0$. 
In [10], we prove:

Theorem 2: Let $\mathcal{M} \in \mathbb{R}^{n \times n}$ be a Metzler matrix and $\Delta$ be a function satisfying Assumption 2. Choose the functions

$$
\begin{aligned}
& \bar{\Phi}_{\mathcal{M}, \bar{\Delta}}(\ell)=\frac{e^{(\mathcal{M}-\bar{\Delta}) \ell}+e^{(\mathcal{M}+\bar{\Delta}) \ell}}{2} \text { and } \\
& \underline{\Phi}_{\mathcal{M}, \bar{\Delta}}(\ell)=e^{\mathcal{M} \ell}+\frac{e^{(\mathcal{M}-\bar{\Delta}) \ell}-e^{(\mathcal{M}+\bar{\Delta}) \ell}}{2} .
\end{aligned}
$$

Then

$$
\underline{\Phi}_{\mathcal{M}, \bar{\Delta}}\left(t-t_{0}\right) \leq \Phi_{\mathcal{M}-\Delta}\left(t, t_{0}\right) \leq \bar{\Phi}_{\mathcal{M}, \bar{\Delta}}\left(t-t_{0}\right)
$$

hold for all $t \geq t_{0}$ and $t_{0} \geq 0$.

Remark 3: In the particular case where $\Delta$ is a constant function, i.e., $\Delta(t)=\Delta_{c}$ for all $t \geq 0$, then $\Phi_{\mathcal{M}-\Delta}\left(t, t_{0}\right)=e^{\left(\mathcal{M}-\Delta_{c}\right)\left(t-t_{0}\right)}$. Also, for any square matrices $\mathcal{A}$ and $\mathcal{B}$ of the same dimension, the inequality

$$
\left\|e^{\mathcal{A}+\mathcal{B}}-e^{\mathcal{A}}\right\| \leq\|\mathcal{B}\| e^{\sup _{\ell \in[0,1]}\|\mathcal{A}+\ell \mathcal{B}\|}
$$

is satisfied, which follows by applying the Fundamental Theorem of Calculus to the function $G(\ell)=e^{\mathcal{A}+\ell \mathcal{B}}$ on the interval $[0,1]$. Taking $\Delta=\Delta_{c}$, it follows from (10) that in this constant $\Delta$ case,

$$
\begin{aligned}
& \left\|\Phi_{\mathcal{M}-\Delta}\left(t, t_{0}\right)-\Phi_{\mathcal{M}}\left(t, t_{0}\right)\right\| \\
& \leq\left(t-t_{0}\right)\left\|\Delta_{c}\right\| e^{\left(t-t_{0}\right) \sup _{\ell \in[-1,1]}\left\|\mathcal{M}+\ell \Delta_{c}\right\|}
\end{aligned}
$$

for all $t \geq t_{0} \geq 0$. On the other hand (9) gives

$$
\underline{\varrho}\left(t-t_{0}\right) \leq \Phi_{\mathcal{M}-\Delta}\left(t, t_{0}\right)-\Phi_{\mathcal{M}}\left(t, t_{0}\right) \leq \bar{\varrho}\left(t-t_{0}\right)
$$

where

$$
\begin{aligned}
& \bar{\varrho}(\ell)=\frac{e^{\left(\mathcal{M}-\Delta_{c}\right) \ell}-e^{\mathcal{M} \ell}+e^{\left(\mathcal{M}+\Delta_{c}\right) \ell}-e^{\mathcal{M} \ell}}{2} \text { and } \\
& \underline{\varrho}(\ell)=\frac{e^{\left(\mathcal{M}-\Delta_{c}\right) \ell}-e^{\mathcal{M} \ell}+e^{\mathcal{M} \ell}-e^{\left(\mathcal{M}+\Delta_{c}\right) \ell}}{2}
\end{aligned}
$$

which gives (11), by (10). The main advantage of Theorem 2 in this time invariant case (over the estimates that can be obtained diredctly from (10)) is that it provides estimates for each entry of $\Phi_{\mathcal{M}-\Delta}\left(t, t_{0}\right)$; see Section VI for an example illustrating this advantage.

\section{B. More General Case}

A limitation of Theorem 2 is that it only applies when $\mathcal{M}$ is Metzler. In this subsection, we relax this Metzler condition by considering the broader family of matrices that are similar to Metzler matrices. This is motivated in part because Jordan canonical forms of matrices with real eigenvalues are Metzler. Also, using the theorem from this subsection, we can relax the Metzler condition from Assumption 1 to only requiring the matrix $M$ in (2) to be similar to a Metzler matrix. In [10], we prove:

Theorem 3: Let $A \in \mathbb{R}^{n \times n}$ admit an invertible matrix $P \in \mathbb{R}^{n \times n}$ and a Metzler matrix $F \in \mathbb{R}^{n \times n}$ such that $P A R=F$, where $R=P^{-1}$. Let $\rho:[0,+\infty) \rightarrow \mathbb{R}^{n \times n}$ and the matrices $\bar{\rho} \in \mathbb{R}^{n \times n}$ and $\underline{\rho} \in \mathbb{R}^{n \times n}$ be such that

$$
\underline{\rho} \leq \rho(t) \leq \bar{\rho}
$$

for all $t \geq 0$. Choose the functions

$$
\begin{aligned}
& \Phi_{£}(\ell)=\frac{R^{+}}{2}\left(e^{(M-\tilde{\xi}) \ell}+e^{(M+\tilde{\kappa}) \ell}\right) P^{+} \\
& -R^{-}\left(e^{M \ell}+\frac{e^{(M-\tilde{\xi}) \ell}-e^{(M+\tilde{\xi}) \ell}}{2}\right) P^{+} \\
& -R^{+}\left(e^{M \ell}+\frac{e^{(M-\tilde{\xi}) \ell}-e^{(M+\tilde{\xi}) \ell}}{2}\right) P^{-} \\
& +\frac{R^{-}}{2}\left(e^{(M-\tilde{\varsigma}) \ell}+e^{(M+\tilde{\varsigma}) \ell}\right) P^{-} \\
& \Phi_{*}(\ell)=R^{+}\left(e^{M \ell}+\frac{e^{(M-\tilde{\xi}) \ell}-e^{(M+\tilde{\xi}) \ell}}{2}\right) P^{+} \\
& -\frac{R^{-}}{2}\left(e^{(M-\tilde{\varsigma}) \ell}+e^{(M+\tilde{\varsigma}) \ell}\right) P^{+} \\
& -\frac{R^{+}}{2}\left(e^{(M-\tilde{\varsigma}) \ell}+e^{(M+\tilde{\varsigma}) \ell}\right) P^{-} \\
& +R^{-}\left(e^{M \ell}+\frac{e^{(M-\tilde{\xi}) \ell}-e^{(M+\tilde{\xi}) \ell}}{2}\right) P^{-}
\end{aligned}
$$

where

$\tilde{\varsigma}=$

$\left(\left(P^{+} \bar{\rho}-P^{-} \underline{\rho}\right) R^{+}-\left(P^{+} \underline{\rho}-P^{-} \bar{\rho}\right) R^{-}\right)^{+}$

$$
-\left(P^{+} \underline{\rho}-P^{-} \bar{\rho}\right) R^{+}+\left(P^{+} \bar{\rho}-P^{-} \underline{\rho}\right) R^{-}
$$

and

$$
\begin{aligned}
M= & F+\left(\left(P^{+} \bar{\rho}-P^{-} \rho\right) R^{+}\right. \\
& \left.+\left(P^{-} \bar{\rho}-P^{+} \underline{\rho}\right) R^{-}\right)^{+} .
\end{aligned}
$$

Then the inequalities

$$
\Phi_{*}\left(t-t_{0}\right) \leq \Phi_{A+\rho}\left(t, t_{0}\right) \leq \Phi_{£}\left(t-t_{0}\right)
$$

hold for all $t \geq t_{0}$ and $t_{0} \geq 0$.

\section{Proof of Theorem 1}

We use Theorem 2 to prove Theorem 1. The proof has three main parts. In the first part, we derive a sample data system that corresponds to the closed loop system from Theorem 1, which is the dynamics for the discrete time variables $x_{k}=X\left(t_{k}\right)$. In the second part, we prove an ISS property of this discrete time system, using the Schur stability assumption from the theorem. In the final part, we use the ISS property for the discrete time system to obtain an ISS property for the closed loop system from the statement of the theorem.

First Step: Deriving the Discrete Time System. Let $i \geq 0$ be an integer. By integrating the system (2) over the interval $\left[t_{i}, t\right]$, with $t \in\left[t_{i}, t_{i+1}\right]$, we obtain

$$
\begin{aligned}
X(t)= & \Phi_{M-\delta_{1}}\left(t, t_{i}\right) X\left(t_{i}\right) \\
& +\int_{t_{i}}^{t} \Phi_{M-\delta_{1}}(t, \ell) B U(\ell) \mathrm{d} \ell \\
& +\int_{t_{i}}^{t} \Phi_{M-\delta_{1}}(t, \ell) \delta_{2}(\ell) \mathrm{d} \ell .
\end{aligned}
$$

Consequently, since $M$ is constant, we get

$$
\begin{aligned}
X(t) & =\left\{e^{M\left(t-t_{i}\right)}\left[X\left(t_{i}\right)+\int_{t_{i}}^{t} e^{M\left(t_{i}-\ell\right)} B U(\ell) \mathrm{d} \ell\right]\right\} \\
& +\int_{t_{i}}^{t} \Phi_{M-\delta_{1}}(t, \ell) \delta_{2}(\ell) \mathrm{d} \ell \\
& +\left[\Phi_{M-\delta_{1}}\left(t, t_{i}\right)-\Phi_{M}\left(t, t_{i}\right)\right] X\left(t_{i}\right) \\
& +\int_{t_{i}}^{t}\left[\Phi_{M-\delta_{1}}(t, \ell)-\Phi_{M}(t, \ell)\right] B U(\ell) \mathrm{d} \ell,
\end{aligned}
$$


since $\Phi_{M}(t, \ell)=e^{M(t-\ell)}$ for all $\ell \in\left[t_{i}, t\right]$. Then the feedback (6) gives

$$
\begin{aligned}
X(t)= & \int_{t_{i}}^{t} \Phi_{M-\delta_{1}}(t, \ell) \delta_{2}(\ell) \mathrm{d} \ell+\mathcal{D}\left(t, t_{i}\right) \\
& +\left[\Phi_{M-\delta_{1}}\left(t, t_{i}\right)-\Phi_{M}\left(t, t_{i}\right)\right] X\left(t_{i}\right) \\
& +\int_{t_{i}}^{t}\left[\Phi_{M}(t, \ell)-\Phi_{M-\delta_{1}}(t, \ell)\right] S_{\ell}^{\sharp} \mathrm{d} \ell X\left(t_{i}\right) \\
= & {\left.\left[-\int_{t_{i}}^{t} \Phi_{D}(t, \ell)\right) S_{\ell}^{\sharp} \mathrm{d} \ell+\Phi_{D}\left(t, t_{i}\right)\right] X\left(t_{i}\right) } \\
& +\int_{t_{i}}^{t} \Phi_{M-\delta_{1}}(t, \ell) \delta_{2}(\ell) \mathrm{d} \ell+\mathcal{D}\left(t, t_{i}\right),
\end{aligned}
$$

where $\mathcal{D}\left(t, t_{i}\right)$ is the quantity in curly braces in (20),

$$
S_{\ell}^{\sharp}=S e^{-M^{\top}(\ell-\sigma(\ell))} J^{-1}\left(r_{i}\right),
$$

$r_{i}=t_{i+1}-t_{i}$, and $\Phi_{D}(a, b)=\Phi_{M-\delta_{1}}(a, b)-\Phi_{M}(a, b)$. Consequently,

$$
\begin{aligned}
X\left(t_{i+1}\right)= & \mathcal{F}(i) X\left(t_{i}\right) \\
& +\int_{t_{i}}^{t_{i+1}} \Phi_{M-\delta_{1}}\left(t_{i+1}, \ell\right) \delta_{2}(\ell) \mathrm{d} \ell,
\end{aligned}
$$

where

$$
\mathcal{F}(i)=\Phi_{D}\left(t_{i+1}, t_{i}\right)-\int_{t_{i}}^{t_{i+1}} \Phi_{D}\left(t_{i+1}, \ell\right) S_{\ell}^{\sharp} \mathrm{d} \ell,
$$

and where we also used the fact that our formula for our control $U$ from (6) and our choice of $J$ in (4) give $\mathcal{D}\left(t_{i+1}, t_{i}\right)=0$. Equation (23) defines the desired closed loop discrete time system.

Second Step: Stability Analysis for Discrete Time System (23). Theorem 2 ensures that for all $a \geq b$, we have

$$
\underline{\xi}(a-b) \leq \Phi_{D}(a, b) \leq \bar{\xi}(a-b),
$$

where $\bar{\xi}$ and $\underline{\xi}$ are defined in (4). We deduce that, for any integer $i \geq 0$, we have

$$
\begin{aligned}
& \underline{\xi}\left(t_{i+1}-\ell\right)\left(S e^{M^{\top}(\sigma(\ell)-\ell)} J^{-1}\left(r_{i}\right)\right)^{+} \\
& \leq \Phi_{D}\left(t_{i+1}, \ell\right)\left(S e^{M^{\top}(\sigma(\ell)-\ell)} J^{-1}\left(r_{i}\right)\right)^{+} \\
& \leq \bar{\xi}\left(t_{i+1}-\ell\right)\left(S e^{M^{\top}(\sigma(\ell)-\ell)} J^{-1}\left(r_{i}\right)\right)^{+}
\end{aligned}
$$

and

$$
\begin{aligned}
& \underline{\xi}\left(t_{i+1}-\ell\right)\left(S e^{M^{\top}(\sigma(\ell)-\ell)} J^{-1}\left(r_{i}\right)\right)^{-} \\
& \leq \Phi_{D}\left(t_{i+1}, \ell\right)\left(S e^{M^{\top}(\sigma(\ell)-\ell)} J^{-1}\left(r_{i}\right)\right)^{-} \\
& \leq \bar{\xi}\left(t_{i+1}-\ell\right)\left(S e^{M^{\top}(\sigma(\ell)-\ell)} J^{-1}\left(r_{i}\right)\right)^{-}
\end{aligned}
$$

for all $\ell \in\left(t_{i}, t_{i+1}\right)$. By multiplying (27) through by -1 and then adding the result to (26), we get

$$
\begin{aligned}
& \underline{\xi}\left(t_{i+1}-\ell\right) \hat{S}_{i, \ell}^{+}-\bar{\xi}\left(t_{i+1}-\ell\right) \hat{S}_{i, \ell}^{-} \\
& \leq \Phi_{D}\left(t_{i+1}, \ell\right) \hat{S}_{i, \ell} \\
& \leq \bar{\xi}\left(t_{i+1}-\ell\right) \hat{S}_{i, \ell}^{+}-\underline{\xi}\left(t_{i+1}-\ell\right) \hat{S}_{i, \ell}^{-}
\end{aligned}
$$

for all $\ell \in\left(t_{i}, t_{i+1}\right)$, where

$$
\hat{S}_{i, \ell}=S e^{M^{\top}\left(t_{i}-\ell\right)} J^{-1}\left(r_{i}\right) .
$$

We deduce that $\underline{\mathcal{F}}(i) \leq \mathcal{F}(i) \leq \overline{\mathcal{F}}(i)$, with $\overline{\mathcal{F}}(i)$ and $\underline{\mathcal{F}}(i)$ defined as follows:

$$
\begin{aligned}
& \overline{\mathcal{F}}(i)=\bar{\xi}\left(t_{i+1}-t_{i}\right) \\
& +\int_{t_{i}}^{t_{i+1}}\left[\bar{\xi}\left(t_{i+1}-\ell\right) \hat{S}_{i, \ell}^{-}-\underline{\xi}\left(t_{i+1}-\ell\right) \hat{S}_{i, \ell}^{+}\right] \mathrm{d} \ell
\end{aligned}
$$

and

$$
\begin{aligned}
& \underline{\mathcal{F}}(i)=\underline{\xi}\left(t_{i+1}-t_{i}\right) \\
& +\int_{t_{i}}^{t_{i+1}}\left[\underline{\xi}\left(t_{i+1}-\ell\right) \hat{S}_{i, \ell}^{-}-\bar{\xi}\left(t_{i+1}-\ell\right) \hat{S}_{i, \ell}^{+}\right] \mathrm{d} \ell .
\end{aligned}
$$

From the definition of the sequence $t_{i}$, we deduce that for each $i \geq 0$, we have $\overline{\mathcal{F}}(i)=\overline{\mathcal{F}}_{c}\left(r_{i}\right)$ and $\underline{\mathcal{F}}(i)=$ $\underline{\mathcal{F}}_{c}\left(r_{i}\right)$ with $\overline{\mathcal{F}}_{c}$ and $\underline{\mathcal{F}}_{c}$ defined in (5) and $r_{i}=t_{i+1}-t_{i}$ as before. It follows that for all integers $i \geq 0$, we have

$$
\underline{\mathcal{F}}_{c}\left(r_{i}\right) \leq \mathcal{F}(i) \leq \overline{\mathcal{F}}_{c}\left(r_{i}\right)
$$

In order to prove our ISS property for (23), we first study the system

$$
Z\left(t_{i+1}\right)=\mathcal{F}(i) Z\left(t_{i}\right)
$$

that corresponds to the $\delta_{2}=0$ case in (23). By rewriting (33) as $Z\left(t_{i+1}\right)=\left(\mathcal{F}(i)^{+}-\mathcal{F}(i)^{-}\right) Z\left(t_{i}\right)$, we conclude that for any solution $Z\left(t_{i}\right)$ of (33), the function $\Xi\left(t_{i}\right)=$ $\left[\left(Z\left(t_{i}\right)\right)^{\top},\left(-Z\left(t_{i}\right)\right)^{\top}\right]^{\top}$ is a solution of

$$
\left\{\begin{aligned}
\zeta\left(t_{i+1}\right) & =\mathcal{F}(i)^{+} \zeta\left(t_{i}\right)+\mathcal{F}(i)^{-} \psi\left(t_{i}\right) \\
\psi\left(t_{i+1}\right) & =\mathcal{F}(i)^{-} \zeta\left(t_{i}\right)+\mathcal{F}(i)^{+} \psi\left(t_{i}\right) .
\end{aligned}\right.
$$

Moreover, (34) is a positive system.

Next, let $\left[\left(\zeta_{p}\left(t_{i}\right)\right)^{\top},\left(\psi_{p}\left(t_{i}\right)\right)^{\top}\right]^{\top} \quad$ and $\left[\left(\zeta_{n}\left(t_{i}\right)\right)^{\top},\left(\psi_{n}\left(t_{i}\right)\right)^{\top}\right]^{\top}$ denote the solutions of (34) with the initial conditions

$$
\left(\begin{array}{c}
\zeta_{p}\left(t_{0}\right) \\
\psi_{p}\left(t_{0}\right)
\end{array}\right)=\left(\begin{array}{c}
Z\left(t_{0}\right)^{+} \\
Z\left(t_{0}\right)^{-}
\end{array}\right)
$$

and

$$
\left(\begin{array}{c}
\zeta_{n}\left(t_{0}\right) \\
\psi_{n}\left(t_{0}\right)
\end{array}\right)=\left(\begin{array}{c}
Z\left(t_{0}\right)^{-} \\
Z\left(t_{0}\right)^{+}
\end{array}\right)
$$

respectively. Then, noticing that

$$
\begin{aligned}
\Xi\left(t_{0}\right) & =\left(\begin{array}{c}
Z\left(t_{0}\right)^{+}-Z\left(t_{0}\right)^{-} \\
Z\left(t_{0}\right)^{-}-Z\left(t_{0}\right)^{+}
\end{array}\right) \\
& =\left(\begin{array}{c}
\zeta_{p}\left(t_{0}\right) \\
\psi_{p}\left(t_{0}\right)
\end{array}\right)-\left(\begin{array}{c}
\zeta_{n}\left(t_{0}\right) \\
\psi_{n}\left(t_{0}\right)
\end{array}\right),
\end{aligned}
$$

we deduce from the uniqueness of solutions property for (34) that

$$
\Xi\left(t_{i}\right)=\left(\begin{array}{c}
\zeta_{p}\left(t_{i}\right) \\
\psi_{p}\left(t_{i}\right)
\end{array}\right)-\left(\begin{array}{c}
\zeta_{n}\left(t_{i}\right) \\
\psi_{n}\left(t_{i}\right)
\end{array}\right)
$$

for all integers $i \geq 0$. 
Next consider the system

$$
\left\{\begin{aligned}
\bar{\zeta}\left(t_{i+1}\right) & =\overline{\mathcal{F}}_{c}^{+}\left(r_{i}\right) \bar{\zeta}\left(t_{i}\right)+\underline{\mathcal{F}}_{c}^{-}\left(r_{i}\right) \bar{\psi}\left(t_{i}\right) \\
\bar{\psi}\left(t_{i+1}\right) & =\underline{\mathcal{F}}_{c}^{-}\left(r_{i}\right) \bar{\zeta}\left(t_{i}\right)+\overline{\mathcal{F}}_{c}^{+}\left(r_{i}\right) \bar{\psi}\left(t_{i}\right) .
\end{aligned}\right.
$$

Let $\left[\left(\bar{\zeta}_{p}\left(t_{i}\right)\right)^{\top},\left(\bar{\psi}_{p}\left(t_{i}\right)\right)^{\top}\right]^{\top}$ and $\left[\left(\bar{\zeta}_{n}\left(t_{i}\right)\right)^{\top},\left(\bar{\psi}_{n}\left(t_{i}\right)\right)^{\top}\right]^{\top}$ denote the solutions of (39) satisfying

$$
\begin{aligned}
& \left(\begin{array}{l}
\bar{\zeta}_{p}\left(t_{0}\right) \\
\bar{\psi}_{p}\left(t_{0}\right)
\end{array}\right)=\left(\begin{array}{c}
Z\left(t_{0}\right)^{+} \\
Z\left(t_{0}\right)^{-}
\end{array}\right) \text {and } \\
& \left(\begin{array}{c}
\bar{\zeta}_{n}\left(t_{0}\right) \\
\bar{\psi}_{n}\left(t_{0}\right)
\end{array}\right)=\left(\begin{array}{c}
Z\left(t_{0}\right)^{-} \\
Z\left(t_{0}\right)^{+}
\end{array}\right)
\end{aligned}
$$

respectively. Then, by the order preserving properties from Section II and the comparison principle, we have

$$
\begin{aligned}
& 0 \leq\left(\begin{array}{c}
\zeta_{p}\left(t_{i}\right) \\
\psi_{p}\left(t_{i}\right)
\end{array}\right) \leq\left(\begin{array}{c}
\bar{\zeta}_{p}\left(t_{i}\right) \\
\bar{\psi}_{p}\left(t_{i}\right)
\end{array}\right) \text { and } \\
& 0 \leq\left(\begin{array}{c}
\zeta_{n}\left(t_{i}\right) \\
\psi_{n}\left(t_{i}\right)
\end{array}\right) \leq\left(\begin{array}{c}
\bar{\zeta}_{n}\left(t_{i}\right) \\
\bar{\psi}_{n}\left(t_{i}\right)
\end{array}\right)
\end{aligned}
$$

for all $i \geq 0$. As an immediate consequence,

$$
\begin{aligned}
-\Gamma^{i}\left(\begin{array}{c}
\bar{\zeta}_{n}\left(t_{0}\right) \\
\bar{\psi}_{n}\left(t_{0}\right)
\end{array}\right) & \leq-\left(\begin{array}{c}
\bar{\zeta}_{n}\left(t_{i}\right) \\
\bar{\psi}_{n}\left(t_{i}\right)
\end{array}\right) \\
& \leq \Xi\left(t_{i}\right) \\
& \leq\left(\begin{array}{c}
\bar{\zeta}_{p}\left(t_{i}\right) \\
\bar{\psi}_{p}\left(t_{i}\right)
\end{array}\right) \\
& \leq \Gamma^{i}\left(\begin{array}{c}
\bar{\zeta}_{p}\left(t_{0}\right) \\
\bar{\psi}_{p}\left(t_{0}\right)
\end{array}\right)
\end{aligned}
$$

for all $i \geq 0$, where $\Gamma$ is the Schur stable matrix from (7), and where we used (38), (41), and the nonnegativity of the terms in the difference in (38). Consequently,

$$
\begin{aligned}
-\Gamma^{i}\left(\begin{array}{c}
Z\left(t_{0}\right)^{-} \\
Z\left(t_{0}\right)^{+}
\end{array}\right) & \leq\left(\begin{array}{c}
Z\left(t_{i}\right) \\
-Z\left(t_{i}\right)
\end{array}\right) \\
& \leq \Gamma^{i}\left(\begin{array}{c}
Z\left(t_{0}\right)^{+} \\
Z\left(t_{0}\right)^{-}
\end{array}\right),
\end{aligned}
$$

by our choice of $\Xi$. Thus,

$$
\begin{aligned}
-[I, 0] \Gamma^{i}\left(\begin{array}{c}
Z\left(t_{0}\right)^{-} \\
Z\left(t_{0}\right)^{+}
\end{array}\right) & \leq Z\left(t_{i}\right) \\
& \leq[I, 0] \Gamma^{i}\left(\begin{array}{c}
Z\left(t_{0}\right)^{+} \\
Z\left(t_{0}\right)^{-}
\end{array}\right) .
\end{aligned}
$$

By our assumption that $\Gamma$ is Schur stable, this implies exponential stability of (33), hence the desired ISS property of (23), by the boundedness of $\delta_{1}$ (which can be shown, e.g., by showing that the strict Lyapunov function for (33) that can be constructed from [2, Theorem $5.13]$ is an ISS Lyapunov function for (23)).

Third Step: ISS Conclusion of Theorem 1. By applying the method of variation of parameters to (2) and recalling our control formula (6), we can find a constant $\bar{c}>0$ such that all solutions of the closed loop system satisfy

$$
|X(t)| \leq \bar{c}\left(|X(\sigma(t))|+\sup \left\{\left|\delta_{2}(\ell)\right|: 0 \leq \ell \leq t\right\}\right)
$$

for all $t \geq 0$. We can combine this with the ISS conclusion from the second step to obtain the ISS property of the closed loop system from the theorem. This proves Theorem 1.

\section{ApPLICATIONS}

In the important special case where the additive disturbance $\delta_{2}$ in (2) is zero, Theorem 1 ensures that the closed loop system with the control (6) is uniformly globally exponentially stable to the origin on $\mathbb{R}^{n}$ when the matrix (7) is Schur stable. On the other hand, we will see in this section that we can also use (10) to get an alternative sufficient condition for this exponential stability property. In the following example, we show that our Schur stability condition from Theorem 1 is less restrictive than the condition that would arise from (10), by showing that our Schur condition holds under larger bounds $\bar{\delta}$ on $\delta_{1}$ than the largest bound that would be allowed if we had instead used (10). This will show an advantage of our Schur stability condition. Although we assume in this section that $\delta_{2}=0$ and that $t_{i}=i \nu$ for all $i \geq 0$ and that the matrix $\delta_{1}$ is a constant matrix $\bar{\delta}$, similar reasoning applies for time varying $\delta_{1}$ 's and for ISS cases where $\delta_{2}$ is not the zero function.

Consider the system (2) with

$$
\begin{aligned}
& M=I \in \mathbb{R}^{2 \times 2}, B=I \in \mathbb{R}^{2 \times 2}, \\
& \text { and } \bar{\delta}=\left[\begin{array}{cc}
0 & \delta_{*} \\
\delta_{*} & 0
\end{array}\right] \in \mathbb{R}^{2 \times 2}
\end{aligned}
$$

with $\delta_{*} \in(0,2)$. Then $S=I$ and

$$
J(r)=\int_{0}^{r} e^{-2 \ell} \mathrm{d} \ell I=\frac{1}{2}\left(1-e^{-2 r}\right) I .
$$

Moreover, since $e^{(M \pm \bar{\delta}) \ell}=e^{M \ell} e^{ \pm \bar{\delta} \ell}$, the relations

$$
e^{(M+\bar{\delta}) \ell}=\left[\begin{array}{ll}
\frac{e^{\left(1+\delta_{*}\right) \ell}+e^{\left(1-\delta_{*}\right) \ell}}{2} & \frac{e^{\left(1+\delta_{*}\right) \ell}-e^{\left(1-\delta_{*}\right) \ell}}{2} \\
\frac{e^{\left(1+\delta_{*}\right) \ell}-e^{\left(1-\delta_{*}\right) \ell}}{2} & \frac{e^{\left(1+\delta_{*}\right) \ell}+e^{\left(1-\delta_{*}\right) \ell}}{2}
\end{array}\right]
$$

and

$$
e^{(M-\bar{\delta}) \ell}=\left[\begin{array}{ll}
\frac{e^{\left(1-\delta_{*}\right) \ell}+e^{\left(1+\delta_{*}\right) \ell}}{2} & \frac{e^{\left(1-\delta_{*}\right) \ell}-e^{\left(1+\delta_{*}\right) \ell}}{2} \\
\frac{e^{\left(1-\delta_{*}\right) \ell}-e^{\left(1+\delta_{*}\right) \ell}}{2} & \frac{e^{\left(1-\delta_{*}\right) \ell}+e^{\left(1+\delta_{*}\right) \ell}}{2}
\end{array}\right]
$$

hold. Consequently, the functions $\bar{\xi}$ and $\underline{\xi}$ from (4) are

$$
\begin{aligned}
& \bar{\xi}(\ell)=e^{\ell}\left(\cosh \left(\delta_{*} \ell\right)-1\right) I \\
& \text { and } \underline{\xi}(\ell)=-e^{\ell} \sinh \left(\delta_{*} \ell\right)\left[\begin{array}{ll}
0 & 1 \\
1 & 0
\end{array}\right],
\end{aligned}
$$

and the functions from (5) are

$$
\begin{aligned}
& \overline{\mathcal{F}}_{c}(r)=e^{r}\left(\cosh \left(\delta_{*} r\right)-1\right) I \\
& +\frac{2}{1-e^{-2 r}} \int_{0}^{r} e^{r-2 \ell} \sinh \left(\delta_{*}(r-\ell)\right) \mathrm{d} \ell\left[\begin{array}{ll}
0 & 1 \\
1 & 0
\end{array}\right] \\
& \quad \text { and } \underline{\mathcal{F}}_{c}(r)=-e^{r} \sinh \left(\delta_{*} r\right)\left[\begin{array}{cc}
0 & 1 \\
1 & 0
\end{array}\right] \\
& \quad-\frac{2}{1-e^{-2 r}} \int_{0}^{r} e^{r-2 \ell}\left[\cosh \left(\delta_{*}(r-\ell)\right)-1\right] \mathrm{d} \ell I,
\end{aligned}
$$


because $\left(S e^{-M^{\top} \ell} J^{-1}\right)^{-}$is the zero matrix. Since $\cosh (s) \geq 1$ holds for all $s \in \mathbb{R}$, it follows that in this example, we have $\overline{\mathcal{F}}_{c}^{+}=\overline{\mathcal{F}}_{c}$ and $\underline{\mathcal{F}}_{c}^{-}=-\underline{\mathcal{F}}_{c}$. With the preceding choices of $\overline{\mathcal{F}}_{c}^{+}$and $\underline{\mathcal{F}}_{c}^{-}$, the closed loop system from Theorem 1 will be globally exponentially stable to 0 on $\mathbb{R}^{n}$ if the $\Gamma$ from (7) is Schur stable.

Next, observe that in this case, the function (24) is

$$
\begin{aligned}
\mathcal{F}(i)= & -\int_{t_{i}}^{t_{i+1}} \mathcal{G}_{3}\left(t_{i+1}, \ell\right) e^{-\ell+\sigma(\ell)} \mathrm{d} \ell \frac{2}{1-e^{-2 \nu}} I \\
& +\mathcal{G}_{3}\left(t_{i+1}, t_{i}\right),
\end{aligned}
$$

where $\mathcal{G}_{3}(t, \ell)=\Phi_{M-\delta_{1}}(t, \ell)-\Phi_{M}(t, \ell)$. Then, in the special case where $\delta_{1}=\bar{\delta}$, we have

$$
\begin{aligned}
& \mathcal{F}(i)=e^{\left(t_{i+1}-t_{i}\right)(I-\bar{\delta})}-e^{\left(t_{i+1}-t_{i}\right) I} \\
& -\frac{2}{1-e^{-2 \nu}} \int_{t_{i}}^{t_{i+1}}\left[e^{\left(t_{i+1}-\ell\right)(I-\bar{\delta})}-e^{\left(t_{i+1}-\ell\right) I}\right] e^{t_{i}-\ell} \mathrm{d} \ell
\end{aligned}
$$

and so also

$$
\begin{aligned}
& \mathcal{F}(i)=e^{\nu(I-\bar{\delta})}-e^{\nu I} \\
& -\frac{2}{1-e^{-2 \nu}} \int_{0}^{\nu}\left[e^{(\nu-\ell)(I-\bar{\delta})}-e^{(\nu-\ell) I}\right] e^{-\ell} \mathrm{d} \ell I .
\end{aligned}
$$

Then, from (10), we deduce that

$$
\begin{aligned}
& \|\mathcal{F}(i)\| \leq \nu^{\sharp} \int_{0}^{\nu}(\nu-\ell)\|\bar{\delta}\| e^{(\nu-\ell)(1+\| \bar{\delta}||)} e^{-\ell} \mathrm{d} \ell \\
& +\nu \| \bar{\delta}|| e^{\nu(1+\| \bar{\delta}||)} \\
& =\nu^{\sharp}\|\bar{\delta}\| \int_{0}^{\nu}(\nu-\ell) e^{\nu(1+|| \bar{\delta}||)-\ell(2+|| \bar{\delta}||)} \mathrm{d} \ell \\
& +\nu \| \bar{\delta}|| e^{\nu(1+|| \bar{\delta}||)} \\
& =\nu^{\sharp} \| \bar{\delta}|| e^{\nu(1+|| \bar{\delta}||)}\left[\nu \int_{0}^{\nu} e^{-\ell(2+|| \bar{\delta}||)} \mathrm{d} \ell\right. \\
& \left.-\int_{0}^{\nu} \ell e^{-\ell(2+\| \bar{\delta}||)} \mathrm{d} \ell\right]+\nu\|\bar{\delta}\| e^{\nu(1+\|\bar{\delta}\|)} \\
& =\nu^{\sharp}\|\bar{\delta}\| e^{\nu(1+\| \bar{\delta}||)}\left[\nu \frac{1-e^{-\nu(2+|| \bar{\delta} \mid)}}{2+|| \bar{\delta} \|}\right. \\
& +\frac{e^{-\nu(2+\| \bar{\delta} \mid)} \nu}{2+\|\delta\|} \\
& \left.+\frac{-1+e^{-\nu(2+|| \bar{\delta}||)}}{(2+|| \bar{\delta}||)^{2}}\right]+\nu\|\bar{\delta}\| e^{\nu(1+|| \bar{\delta}||)} \\
& =\nu^{\sharp}\|\bar{\delta}\| e^{\nu(1+\|\bar{\delta}\|)}\left(\frac{\nu}{2+\|\delta\|}\right. \\
& \left.+\frac{-1+e^{-\nu(2+|| \bar{\delta}||)}}{(2+\| \bar{\delta}||)^{2}}\right)+\nu\|\bar{\delta}\| e^{\nu(1+\| \bar{\delta}||)},
\end{aligned}
$$

where $\nu^{\sharp}=\frac{2}{1-e^{-2 \nu}}$. This gives the condition

$$
\begin{aligned}
\|\mathcal{F}(i)\| \leq & \frac{2 \delta_{*} e^{\nu\left(1+\delta_{*}\right)}}{\left(1-e^{-2 \nu}\right)\left(2+\delta_{*}\right)}\left(\nu+\frac{e^{-\nu\left(2+\delta_{*}\right)}-1}{2+\delta_{*}}\right) \\
& +\nu \delta_{*} e^{\nu\left(1+\delta_{*}\right)} .
\end{aligned}
$$

Therefore, by using (10), we conclude that the closed loop system will satisfy the desired exponential stability property if the right side of (56) is in $(0,1)$, by the second part of the proof of Theorem 1. This is a more restrictive condition than our Schur stability condition from Theorem 1, since, e.g., if $\delta_{*}=0.36$ and $\nu=0.72$ in the previous example, then simple Mathematica calculations show that the eigenvalues of the matrix $\Gamma$ from (7) are $\{0.969621,-0.767802,0.246164,-0.170381\}$ (so the Schur stability requirement is satisfied) but the right side of (56) is 1.0879. Hence, Theorem 1 gives a better stability condition than the one that we would have gotten by merely applying (10).

\section{CONCLUSIONS}

We contributed to the literature on feedback stabilization for systems with uncertainty and sampling. We allowed additive uncertainty on the right side of the dynamics, and in a vector field defining the system. Our strategy combined positive systems approaches with new matrix inequalities that estimate fundamental solutions for time-varying linear systems with uncertain coefficients. Due to the difficulty in estimating fundamental matrix solutions for time-varying linear systems with uncertainties, our matrix estimations are of independent interest. Our example illustrated an advantage of using our new stabilization approach. In future work, we aim to extend our work to event-triggered [3] nonlinear systems whose right sides include Metzler matrices and to provide analogs that compensate for input delays [10].

\section{REFERENCES}

[1] K. Astrom and B. Wittenmark. Computer-Controlled Systems Theory and Design. Third Edition. Dover Publications, Mineola, NY, 1997.

[2] N. Bof, R. Carli, and L. Schenato. Lyapunov theory for discrete time systems. 2018, https://arxiv.org/abs/1809.05289.

[3] A. Borri and P. Pepe. Event-triggered control of nonlinear systems with time-varying state delays. IEEE Transactions on Automatic Control, to appear, http://dx.doi.org/10.1109/TAC.2020.3009173

[4] F. Cacace, A. Germani and C. Manes. An enhanced observer for nonlinear systems with time-varying measurement delays. IEEE Transactions on Automatic Control, to appear, http://dx.doi.org/10.1109/TAC.2020.3046723.

[5] J.-L. Gouzé, A. Rapaport, and Z. Hadj-Sadok. Interval observers for uncertain biological systems. Ecological Modelling, 133(12):45-56, 2000.

[6] J. Klamka. Controllability and Minimum Energy Control. Springer, New York, 2019.

[7] M. Mattioni, S. Monaco, and D. Normand-Cyrot. Immersion and invariance stabilization of strict-feedback dynamics under sampling. Automatica, 76:78-86, 2017.

[8] M. Mattioni, S. Monaco, and D. Normand-Cyrot. Feedforwarding under sampling. IEEE Transactions on Automatic Control, 64(11):4668-4675, 2019

[9] F. Mazenc, V. Andrieu, and M. Malisoff. Design of continuousdiscrete observers for time-varying nonlinear systems. Automatica, 57(7):135-144, 2015.

[10] F. Mazenc and M. Malisoff. Feedback stabilization and robustness analysis with discrete measurements using bounds on fundamental matrices. In Preparation. http://www.math.lsu.edu/ malisoff/. For the convenience of the reviewers of this initial submission, we include proofs of Theorems 2-3 from [10] in the appendices below.

[11] T. Raissi, D. Efimov, and A. Zolghadri. Interval state estimation for a class of nonlinear systems. IEEE Transactions on Automatic Control, 57(1):260-265, 2012.

[12] A. Selivanov and E. Fridman. Delayed point control of a reaction-diffusion PDE under discrete-time point measurements. Automatica, 96: 224-233, 2018.

[13] E. Sontag. Mathematical Control Theory, Second Edition. Springer, New York, NY, 1998

[14] E. Sontag. Input to state stability: basic concepts and results. In Nonlinear and Optimal Control Theory, P. Nistri and G. Stefani, Eds. Springer, Berlin Germany, 2008, pp. 163-220.

[15] V. Tanasa, S. Monaco, and D. Normand-Cyrot. Backstepping control under multi-rate sampling. IEEE Transactions on Automatic Control, 61(5):1208-1222, 2016. 
The following 2 pages contain proofs of Theorem 2-3, which are special cases of proofs from [10], and are for review purposes only, and are not part of the paper being proposed for the proceedings. See p.6 for the references.

\section{Appendix A1: Proof of Theorem 2}

We use the following consequence of the uniqueness of solutions property for (1) with $\mathcal{F}=\mathcal{R}$, which is shown by checking that $\mathcal{H}\left(t, t_{0}\right)=\mathcal{L} \Phi_{\mathcal{A}}\left(t, t_{0}\right) \mathcal{L}^{-1}$ satisfies $\mathcal{H}\left(t_{0}, t_{0}\right)=I$ and $\frac{\partial}{\partial t} H\left(t, t_{0}\right)=\mathcal{R} \mathcal{H}\left(t, t_{0}\right)$ for all $t \geq 0$ and $t_{0} \geq 0$ :

Lemma 1: Let $\mathcal{A}:[0,+\infty) \rightarrow \mathbb{R}^{n \times n}$. Let $\mathcal{L} \in \mathbb{R}^{n \times n}$ be an invertible matrix and let $\mathcal{R}=\mathcal{L} \mathcal{A} \mathcal{L}^{-1}$. Then

$$
\Phi_{\mathcal{R}}\left(t, t_{0}\right)=\mathcal{L} \Phi_{\mathcal{A}}\left(t, t_{0}\right) \mathcal{L}^{-1}
$$

for all $t \geq 0$ and $t_{0} \geq 0$.

Returning to the proof of Theorem 2, we choose the function $\Omega: \mathbb{R} \rightarrow \mathbb{R}^{(2 n) \times(2 n)}$ and the matrix $P$ defined by

$$
\begin{aligned}
& \Omega(t)=\left[\begin{array}{cc}
\mathcal{M} & \Delta(t) \\
\Delta(t) & \mathcal{M}
\end{array}\right] \text { and } \\
& \mathcal{P}=\left[\begin{array}{rr}
I & I \\
-I & I
\end{array}\right] \in \mathbb{R}^{(2 n) \times(2 n)} .
\end{aligned}
$$

Elementary calculations give

$$
\begin{aligned}
& \mathcal{P}^{-1}=\left[\begin{array}{rr}
\frac{I}{2} & -\frac{I}{2} \\
\frac{I}{2} & \frac{I}{2}
\end{array}\right] \text { and } \mathcal{P} \Omega(t) \mathcal{P}^{-1}=\Theta(t), \\
& \text { where } \Theta(t)=\left[\begin{array}{cc}
\mathcal{M}+\Delta(t) & 0 \\
0 & \mathcal{M}-\Delta(t)
\end{array}\right] .
\end{aligned}
$$

Lemma 1 ensures that $\Phi_{\Theta}=\mathcal{P} \Phi_{\Omega} \mathcal{P}^{-1}$, which we can combine with (A.4) to get

$$
\begin{aligned}
\Phi_{\mathcal{M}-\Delta} & =\left[\begin{array}{ll}
0 & I
\end{array}\right] \mathcal{P} \Phi_{\Omega} P^{-1}\left[\begin{array}{l}
0 \\
I
\end{array}\right] \\
& =\left[\begin{array}{ll}
-I & I
\end{array}\right] \Phi_{\Omega}\left[\begin{array}{r}
-\frac{I}{2} \\
\frac{I}{2}
\end{array}\right] .
\end{aligned}
$$

Also, Assumption 2 guarantees that the inequalities

$$
\left[\begin{array}{cc}
\mathcal{M} & 0 \\
0 & \mathcal{M}
\end{array}\right] \leq \Omega(t) \leq\left[\begin{array}{cc}
\mathcal{M} & \bar{\Delta} \\
\bar{\Delta} & \mathcal{M}
\end{array}\right]
$$

are satisfied for all $t \geq 0$. Since the matrix $\mathcal{M}$ is Metzler and $\Delta$ is nonnegative, it follows that

$$
\begin{aligned}
& {\left[\begin{array}{cc}
e^{\mathcal{M}\left(t-t_{0}\right)} & 0 \\
0 & e^{\mathcal{M}\left(t-t_{0}\right)}
\end{array}\right]} \\
& \leq \Phi_{\Omega}\left(t, t_{0}\right) \leq e^{\left[\begin{array}{cc}
\mathcal{M} & \bar{\Delta} \\
\bar{\Delta} & \mathcal{M}
\end{array}\right]\left(t-t_{0}\right)}
\end{aligned}
$$

for all $t \geq t_{0}$. To see why (A.7) holds, notice that if $X(t)$ and $Z(t)$ are solutions of $\dot{X}=\Omega(t) X$ and $\dot{Z}=\operatorname{diag}(\mathcal{M}, \mathcal{M}) Z$ respectively (where $\operatorname{diag}(\mathcal{M}, \mathcal{M})$ is the block diagonal matrix with $\mathcal{M}$ as the diagonal blocks) with the same initial state $X\left(t_{0}\right) \geq 0$, then
$\Phi_{\Omega}\left(t, t_{0}\right) \geq 0$ and $Z(t) \geq 0$ hold for all $t \geq t_{0}$ (by the Metzler property of $\mathcal{M}$ and the nonnegative valuedness of $\Delta$, using, for instance, [9, Lemma 1]), and applying variation of parameters to $\dot{X}-\dot{Z}=\Omega(t)(X-Z)+$ $(\Omega(t)-\operatorname{diag}(\mathcal{M}, \mathcal{M})) Z$ gives

$$
\begin{aligned}
& X(t)-Z(t)=\Phi_{\Omega}\left(t, t_{0}\right)\left(X\left(t_{0}\right)-Z\left(t_{0}\right)\right) \\
& +\int_{t_{0}}^{t} \Phi_{\Omega}(t, \ell)(\Omega(\ell)-\operatorname{diag}(\mathcal{M}, \mathcal{M})) Z(\ell) \mathrm{d} \ell \geq 0
\end{aligned}
$$

for $t \geq t_{0} \geq 0$, which gives the first inequality in (A.7) by specializing to cases where $X\left(t_{0}\right)$ is a standard basis vector, and the second inequality in (A.7) follows by applying similar reasoning to

$$
\dot{X}=\left[\begin{array}{ll}
\mathcal{M} & \bar{\Delta} \\
\bar{\Delta} & \mathcal{M}
\end{array}\right] X
$$

and $\dot{Z}=\Omega(t) Z$.

Since

$\left[\begin{array}{ll}\mathcal{M} & \bar{\Delta} \\ \bar{\Delta} & \mathcal{M}\end{array}\right]=\mathcal{P}^{-1}\left[\begin{array}{cc}\mathcal{M}+\bar{\Delta} & 0 \\ 0 & \mathcal{M}-\bar{\Delta}\end{array}\right] \mathcal{P}$,

it follows from Lemma 1 that

$$
\begin{aligned}
& e^{\left[\begin{array}{cc}
\mathcal{M} & \bar{\Delta} \\
\bar{\Delta} & \mathcal{M}
\end{array}\right]{ }_{\left(t-t_{0}\right)}}= \\
& \mathcal{P}^{-1}\left[\begin{array}{cc}
e^{(\mathcal{M}+\bar{\Delta})\left(t-t_{0}\right)} & 0 \\
0 & e^{(\mathcal{M}-\bar{\Delta})\left(t-t_{0}\right)}
\end{array}\right] \mathcal{P} .
\end{aligned}
$$

We deduce from (A.7) and (A.11) that

$$
\begin{aligned}
& {\left[\begin{array}{cc}
e^{\mathcal{M}\left(t-t_{0}\right)} & 0 \\
0 & e^{\mathcal{M}\left(t-t_{0}\right)}
\end{array}\right] \leq \Phi_{\Omega}\left(t, t_{0}\right)} \\
& \leq \mathcal{P}^{-1}\left[\begin{array}{cc}
e^{(\mathcal{M}+\bar{\Delta})\left(t-t_{0}\right)} & 0 \\
0 & e^{(\mathcal{M}-\bar{\Delta})\left(t-t_{0}\right)}
\end{array}\right] \mathcal{P}
\end{aligned}
$$

for $t \geq t_{0} \geq 0$. Consequently,

$$
\begin{aligned}
& {\left[\begin{array}{cc}
e^{\mathcal{M}\left(t-t_{0}\right)} & 0 \\
0 & e^{\mathcal{M}\left(t-t_{0}\right)}
\end{array}\right] \leq} \\
& \Phi_{\Omega}\left(t, t_{0}\right) \leq\left[\begin{array}{ll}
\mathcal{G}_{1}\left(t-t_{0}\right) & \mathcal{G}_{2}\left(t-t_{0}\right) \\
\mathcal{G}_{2}\left(t-t_{0}\right) & \mathcal{G}_{1}\left(t-t_{0}\right)
\end{array}\right]
\end{aligned}
$$

for $t \geq t_{0}$, where

$$
\begin{aligned}
& \mathcal{G}_{1}(s)=\frac{e^{(\mathcal{M}+\bar{\Delta}) s}+e^{(\mathcal{M}-\bar{\Delta}) s}}{2} \text { and } \\
& \mathcal{G}_{2}(s)=\frac{e^{(\mathcal{M}+\bar{\Delta}) s}-e^{(\mathcal{M}-\bar{\Delta}) s}}{2} .
\end{aligned}
$$

By right multiplying the inequalities (A.13) through by $[0, I / 2]^{\top}$ and $[-I / 2,0]^{\top}$, we obtain

$$
\begin{aligned}
& {\left[\begin{array}{c}
0 \\
\frac{e^{\mathcal{M}\left(t-t_{0}\right)}}{2}
\end{array}\right] \leq \Phi_{\Omega}\left(t, t_{0}\right)\left[\begin{array}{c}
0 \\
\frac{I}{2}
\end{array}\right] \leq} \\
& {\left[\begin{array}{l}
\frac{e^{(\mathcal{M}+\bar{\Delta})\left(t-t_{0}\right)}-e^{(\mathcal{M}-\bar{\Delta})\left(t-t_{0}\right)}}{4} \\
\frac{e^{(\mathcal{M}+\bar{\Delta})\left(t-t_{0}\right)}+e^{(\mathcal{M}-\bar{\Delta})\left(t-t_{0}\right)}}{4}
\end{array}\right]} \\
& \text { and }\left[\begin{array}{c}
-\frac{e^{(\mathcal{M}+\bar{\Delta})\left(t-t_{0}\right)}+e^{(\mathcal{M}-\bar{\Delta})\left(t-t_{0}\right)}}{4} \\
\frac{e^{(\mathcal{M}-\bar{\Delta})\left(t-t_{0}\right)}-e^{(\mathcal{M}+\bar{\Delta})\left(t-t_{0}\right)}}{4}
\end{array}\right] \\
& \leq \Phi_{\Omega}\left(t, t_{0}\right)\left[\begin{array}{r}
-\frac{I}{2} \\
0
\end{array}\right] \leq\left[\begin{array}{c}
-\frac{e^{\mathcal{M}\left(t-t_{0}\right)}}{2} \\
0
\end{array}\right] \text {, }
\end{aligned}
$$


since multiplying by $-I / 2$ reverses the inequalities. Adding these inequalities yields

$$
\underline{\beta}\left(t-t_{0}\right) \leq \Phi_{\Omega}\left(t, t_{0}\right)\left[\begin{array}{r}
-\frac{I}{2} \\
\frac{I}{2}
\end{array}\right] \leq \bar{\beta}\left(t-t_{0}\right),
$$

where

$$
\begin{aligned}
& \bar{\beta}(\ell)=\left[\begin{array}{c}
\frac{e^{(\mathcal{M}+\bar{\Delta}) \ell}-e^{(\mathcal{M}-\bar{\Delta}) \ell}}{4}-\frac{e^{\mathcal{M} \ell}}{2} \\
\frac{e^{(\mathcal{M}+\bar{\Delta}) \ell}+e^{(\mathcal{M}-\bar{\Delta}) \ell}}{4}
\end{array}\right] \text { and } \\
& \underline{\beta}(\ell)=\left[\begin{array}{c}
-\frac{e^{(\mathcal{M}+\bar{\Delta}) \ell}+e^{(\mathcal{M}-\bar{\Delta}) \ell}}{4} \\
\frac{e^{\mathcal{M} \ell}}{2}+\frac{e^{(\mathcal{M}-\bar{\Delta}) \ell}-e^{(\mathcal{M}+\bar{\Delta}) \ell}}{4}
\end{array}\right] .
\end{aligned}
$$

By left multiplying the inequalities (A.16) by the matrices $\left[\begin{array}{ll}0 & I\end{array}\right]$ and $\left[\begin{array}{ll}-I & 0\end{array}\right]$, we obtain:

$$
\begin{aligned}
{\left[\begin{array}{ll}
0 & I
\end{array}\right] \underline{\beta}(t) } & \leq\left[\begin{array}{ll}
0 & I
\end{array}\right] \Phi_{\Omega}\left(t, t_{0}\right)\left[\begin{array}{r}
-\frac{I}{2} \\
\frac{I}{2}
\end{array}\right] \\
& \leq\left[\begin{array}{ll}
0 & I
\end{array}\right] \bar{\beta}(t)
\end{aligned}
$$

and

$$
\begin{aligned}
{\left[\begin{array}{ll}
-I & 0
\end{array}\right] \bar{\beta}\left(t-t_{0}\right) } & \leq\left[\begin{array}{ll}
-I & 0
\end{array}\right] \Phi_{\Omega}\left(t, t_{0}\right)\left[\begin{array}{r}
-\frac{I}{2} \\
\frac{I}{2}
\end{array}\right] \\
& \leq\left[\begin{array}{ll}
-I & 0
\end{array}\right] \underline{\beta}\left(t-t_{0}\right) .
\end{aligned}
$$

By adding these inequalities, we obtain

$$
\begin{aligned}
& {\left[\begin{array}{ll}
0 & I
\end{array}\right] \underline{\beta}\left(t-t_{0}\right)+\left[\begin{array}{ll}
-I & 0
\end{array}\right] \bar{\beta}\left(t-t_{0}\right)} \\
& \leq\left[\begin{array}{ll}
-I & I
\end{array}\right] \Phi_{\Omega}\left(t, t_{0}\right)\left[\begin{array}{r}
-\frac{I}{2} \\
\frac{I}{2}
\end{array}\right] \\
& \leq\left[\begin{array}{ll}
0 & I
\end{array}\right] \bar{\beta}\left(t-t_{0}\right)+\left[\begin{array}{ll}
-I & 0
\end{array}\right] \underline{\beta}\left(t-t_{0}\right) .
\end{aligned}
$$

Finally, from the second equality in (A.5), we deduce that $\bar{\Phi}_{\mathcal{M}, \bar{\Delta}}\left(t-t_{0}\right) \leq \Phi_{\mathcal{M}-\Delta}\left(t, t_{0}\right) \leq \underline{\Phi}_{\mathcal{M}, \bar{\Delta}}\left(t-t_{0}\right)$, where $\bar{\Phi}_{\mathcal{M}, \bar{\Delta}}$ and $\underline{\Phi}_{\mathcal{M}, \bar{\Delta}}$ are the functions defined in (8). This allows us to conclude.

\section{Appendix A2: Proof of TheOREM 3}

From (14), it follows that $P^{+} \rho \leq P^{+} \rho \leq P^{+} \bar{\rho}$ and $-P^{-} \bar{\rho} \leq-P^{-} \rho \leq-P^{-} \rho$. We deduce from the formula $P \rho=(P \rho)^{+}-(P \rho)^{-}$that the inequalities

$$
P^{+} \underline{\rho}-P^{-} \bar{\rho} \leq P \rho \leq P^{+} \bar{\rho}-P^{-} \underline{\rho}
$$

are satisfied. From them we deduce that

$$
\begin{aligned}
\left(P^{+} \underline{\rho}-P^{-} \bar{\rho}\right) R^{+} & \leq P \rho R^{+} \\
& \leq\left(P^{+} \bar{\rho}-P^{-} \underline{\rho}\right) R^{+}
\end{aligned}
$$

and

$$
\begin{aligned}
-\left(P^{+} \bar{\rho}-P^{-} \underline{\rho}\right) R^{-} & \leq-P \rho R^{-} \\
& \leq-\left(P^{+} \underline{\rho}-P^{-} \bar{\rho}\right) R^{-} .
\end{aligned}
$$

By adding the inequalities (A.22)-(A.23), we obtain

$$
\underline{\varsigma} \leq P \rho R \leq \bar{\varsigma},
$$

where $\bar{\varsigma}$ and $\varsigma$ are defined as follows:

$$
\begin{aligned}
& \bar{\varsigma}=\left(P^{+} \bar{\rho}-P^{-} \rho\right) R^{+}-\left(P^{+} \rho-P^{-} \bar{\rho}\right) R^{-} \\
& \underline{\varsigma}=\left(P^{+} \underline{\rho}-P^{-} \bar{\rho}\right) R^{+}-\left(P^{+} \bar{\rho}-P^{-} \underline{\rho}\right) R^{-} .
\end{aligned}
$$

Then, since the formula (17) gives $M=F+\bar{\varsigma}^{+}$, we get

$$
P(A+\rho) R=F+P \rho R=M-\Delta
$$

where $M$ is the matrix defined in (17) and $\Delta=\bar{\varsigma}^{+}-$ $P \rho R$. The matrix $M$ is Metzler and the inequalities (A.24) give $0 \leq \Delta \leq \bar{\varsigma}^{+}-\underline{\varsigma}$. Next, Theorem 2 ensures that

$$
\begin{aligned}
& e^{M\left(t-t_{0}\right)}+\frac{e^{\left(M-\bar{\varsigma}^{+}+\underline{\varsigma}\right)\left(t-t_{0}\right)}-e^{\left(M+\bar{\varsigma}^{+}-\varsigma\right)\left(t-t_{0}\right)}}{2} \\
& \leq \Phi_{M-\Delta}\left(t, t_{0}\right) \\
& \leq \frac{e^{\left(M-\bar{\varsigma}^{+}+\varsigma\right)\left(t-t_{0}\right)}+e^{\left(M+\bar{\varsigma}^{+}-\varsigma\right)\left(t-t_{0}\right)}}{2}
\end{aligned}
$$

for all $t \geq t_{0} \geq 0$. According to Lemma 1, these inequalities in combination with (A.26) give

$$
\underline{\mu}\left(t-t_{0}\right) \leq P \Phi_{A+\rho}\left(t, t_{0}\right) R \leq \bar{\mu}\left(t-t_{0}\right)
$$

for all $t \geq t_{0} \geq 0$ with

$$
\begin{aligned}
& \bar{\mu}(\ell)=\frac{e^{\left(M-\bar{\varsigma}^{+}+\varsigma\right) \ell}+e^{\left(M+\bar{\varsigma}^{+}-\underline{\varsigma}\right) \ell}}{2} \text { and } \\
& \underline{\mu}(\ell)=e^{M \ell}+\frac{e^{\left(M-\bar{\varsigma}^{+}+\underline{\varsigma}\right) \ell}-e^{\left(M+\bar{\varsigma}^{+}-\underline{\varsigma}\right) \ell}}{2} .
\end{aligned}
$$

Consequently,

$$
\begin{aligned}
R^{+} \underline{\mu}\left(t-t_{0}\right) & \leq R^{+} P \Phi_{A+\rho}\left(t, t_{0}\right) R \\
& \leq R^{+} \bar{\mu}\left(t-t_{0}\right)
\end{aligned}
$$

and

$$
\begin{aligned}
-R^{-} \bar{\mu}\left(t-t_{0}\right) & \leq-R^{-} P \Phi_{A+\rho}\left(t, t_{0}\right) R \\
& \leq-R^{-} \underline{\mu}\left(t-t_{0}\right) .
\end{aligned}
$$

Adding the inequalities in (A.30)-(A.31) and recalling that $R=P^{-1}$, we get

$$
\begin{aligned}
R^{+} \underline{\mu}\left(t-t_{0}\right)-R^{-} \bar{\mu}\left(t-t_{0}\right) \leq & \Phi_{A+\rho}\left(t, t_{0}\right) R \\
\leq & R^{+} \bar{\mu}\left(t-t_{0}\right) \\
& -R^{-} \underline{\mu}\left(t-t_{0}\right) .
\end{aligned}
$$

From these inequalities, we deduce that

$$
\begin{aligned}
& {\left[R^{+} \underline{\mu}\left(t-t_{0}\right)-R^{-} \bar{\mu}\left(t-t_{0}\right)\right] P^{+}} \\
& \leq \Phi_{A+\rho}\left(t, t_{0}\right) R P^{+} \\
& \leq\left[R^{+} \bar{\mu}\left(t-t_{0}\right)-R^{-} \underline{\mu}\left(t-t_{0}\right)\right] P^{+}
\end{aligned}
$$

and

$$
\begin{aligned}
& -\left[R^{+} \bar{\mu}\left(t-t_{0}\right)-R^{-} \underline{\mu}\left(t-t_{0}\right)\right] P^{-} \\
& \leq-\Phi_{A+\rho}\left(t, t_{0}\right) R P^{-} \\
& \leq-\left[R^{+} \underline{\mu}\left(t-t_{0}\right)-R^{-} \bar{\mu}\left(t-t_{0}\right)\right] P^{-} .
\end{aligned}
$$

This allows us to conclude, by adding the inequalities in (A.33)-(A.34) and recalling that $\tilde{\zeta}=\bar{\zeta}^{+}-\underline{\zeta}$.

The preceding 2 pages consisted of material from [10] and were included for review purposes only, and are not part of the paper that is being proposed for inclusion in the proceedings. See p.6 for the references cited. 